\title{
An Advanced Trust-Based Routing Protocol for Mobile AdHoc Network under Black-hole Attack
}

\author{
Manish Devendra Chawhan ${ }^{1}$, Kishor Damodhar Kulat², Sanjay \\ Babanroa Pokle ${ }^{3}$ and Ausaf Umar Khan ${ }^{4^{*}}$ \\ ${ }^{1}$ Associate Professor, Department of Electronics and Telecommunication \\ Engineering, Yeshwantrao Chavan College of Engineering, India \\ ${ }^{2}$ Professor, Department of Electronics and Communication Engineering, \\ Visvesvaraya National Institute of Technology, India \\ ${ }^{3}$ Professor, Department of Electronics and Communication Engineering, \\ Shri Ramdeobaba College of Engineering and Management, India \\ ${ }^{4 *}$ Research Scholar, Department of Electronics and Telecommunication Engineering, \\ Yeshwantrao Chavan College of Engineering, India
}

\section{ABSTRACT}

Mobile Adhoc Network (Manet) is a group of independent mobile nodes without fix topology and centralized administration. Because of absence of centralized administration, Manet is highly exposed to active as well as passive attacks. The existing routing protocols don't have security provision to protect the Manet against any type of attack. The Black hole attack is an active attack and effectively affects the performance of Manet by pretending itself as the safest and shortest path to the destination. So, there is a need of security provision in the routing protocol to protect the network against such attacks to avoid performance degradation. In this paper, An Advanced Trust-Based Routing Protocol (ATBRP) is proposed and implemented in NS2. The simulation results show that the proposed protocol defend the network from Black hole attacks and achieves better performance in terms of Packet Delivery Ratio (PDR), delay and throughput as compared to Dynamic Source Routing (DSR) protocol.

\section{KEY WORDS: DSR, MANET, PDR, SECURITY MECHANISM AND THROUGHPUT.}

\section{INTRODUCTION}

The wireless ad hoc technology has become a worldwide phenomenon in recent times due to tremendous and fast advancement. Recent wireless ad hoc technologies like MANET, VANET, SPAN etc. have a great contribution to

\section{ARTICLE INFORMATION}

*Corresponding Author: ausafumarkhan@gmail.com

Received 12th Oct 2020 Accepted after revision 24th Dec 2020

Print ISSN: 0974-6455 Online ISSN: 2321-4007 CODEN: BBRCBA

Thomson Reuters ISI Web of Science Clarivate Analytics USA and Crossref Indexed Journal

\section{Clarivate
Analytics}

NAAS Journal Score 2020 (4.31)

A Society of Science and Nature Publication,

Bhopal India 2020. All rights reserved.

Online Contents Available at: http//www.bbrc.in/

Doi: http://dx.doi.org/10.21786/bbrc/13.14/29 the development of infrastructure independent wireless technology. A Manet becomes popular because of no administration cost and fast deployment. In Manet, each mobile node can store, transmit and receive binary data to establish communication within its transmission range. Because of decentralized infrastructure, the mobile nodes can participate or leave the communication network as per their convenience. In Manet, nodes are free to exchange their resources within their transmission range, creating separate spontaneous ad hoc networks.

In large Manet, it is important for each node to recognize its neighboring nodes for secure communication during route discovery process. During route discovery process, the source node broadcasts the route request packet (RREQ) to neighbouring nodes within transmission 
range in search of the shortest route to the destination node. During this process, neighbour node (if it is Black Hole) doesn't further broadcast the RREQ and reply the source node with the route reply (RREP) packet. Once the communication is established, that neighbour node acts as Black hole attack and drops all the data packets generated for destination node. This degrades the performance of network and disrupt the communication. To prevent the Manet from such type of attacks, a security mechanism must be provided in the routing protocol.

Literature Survey: An Evolutionary Self-Cooperative Trust Scheme (ESCT) is proposed to tackle different types of attacks in MANET. The proposed method is intellectually handle the internal attacks and lower the effect of them. This trust based mechanism able to defend a Manet against black hole as well as gray hole attacks. A trust-based DSR protocol is presented in which determines the fast and safe route in cooperation with neighbour node. This method able to protect the Manet without monitoring the neighbouring nodes. The proposed method able to yield better results in the presence of cooperative black hole attack. The new request forwarding mechanism is presented called EM-DSR. This method decreases the communication overhead and delay in the presence of attack. The techniques presented for packet classification used in intrusion detection system and firewall may be effective for Manet. because of dynamic characteristics of Manet, implementing security mechanism is bottleneck.

\section{Network Model and Proposed Work}

a. Network Model: In the proposed protocol, a neighboring node is assigned a trust rating depending on its behavior. First, the activity of the node is examined to adjust the trust level. It is not always that the node drops packets due to its malicious behavior, or it's a black hole attack. Sometimes a node may be a benign node or drops packets due to congestion. Therefore, it is crucial to ensure that the node is dropping the packets intentionally. In this process of judgment, the protocol need to maintain following five types of records:

1. Sent Packet Count.

2. Received packet Count by the neighboring node.

3. Self-assessment rating

4. Mutual-assessment rating.

5. Neighbor judgment.

Before the final assignment of trust rating, it is initialized to zero for all nodes. It will increase or decrease depending upon the sent packet and received packet count. In this work, the final positive trust rating is 40 with the negative trust rating -40 . The sent_count is the count of forwarded packets to a neighbor node, and each node updated this value at a one-hop distance. The received_count is the value of packets received from the source node, and this value has to maintain updated by each neighbor node. To monitor the packet forwarding activity, every node has to further forward the packets within timestamp, otherwise it is assumed that the packet has dropped and a negative event is recorded for that particular node. b. Advanced Trust-Based Routing Protocol (ATBRP): The proposed method includes self assessment and mutual assessment of trust rating to the node. Both assessment helps to find the malicious node or attacks present in the Manet. This proposed mechanism is very effective and achieves better results for the Manet under multiple attacks. In this section, proposed method is illustrated with flowchart.

i.Self-assessment: Each node maintains a record of packets forwarded to neighbor nodes and monitors their activity. If the neighbor drops one packet a negative event is recorded and the trust rating decreases by 2 . This is done by comparing the sent_count and received_count. When the neighbor node forwards the packet, then the positive event is recorded and the trust rating increases by 1 . So, if a node drops packets due to traffic, that instance, it drops the packet and at other instance, it forwards the packets. This process doesn't affect the trust rating of node due to both event. On the other hand, if a node is malicious, then it drops the packets continuously, and repetitive negative events are recorded. If the value of drop packets reaches 20, then the trust rating will reach -40 . At this point, the neighbor node is recognized as malicious and it is added to the black list and the routes containing this node are deleted. The neighbor id with its rating is broadcasted using the HELLO message so that the other nodes must also be aware of that malicious node in their vicinity. A node of rating -40 is abandoned, whereas a node of rating 40 is considered as the most trusted node.

ii. Mutual-assessment: In this type of assessment, each node keeps a record of the trust ratings provided by other nodes to their neighboring node for comparasion. Nodes retrieve their ratings from the broadcast messages.

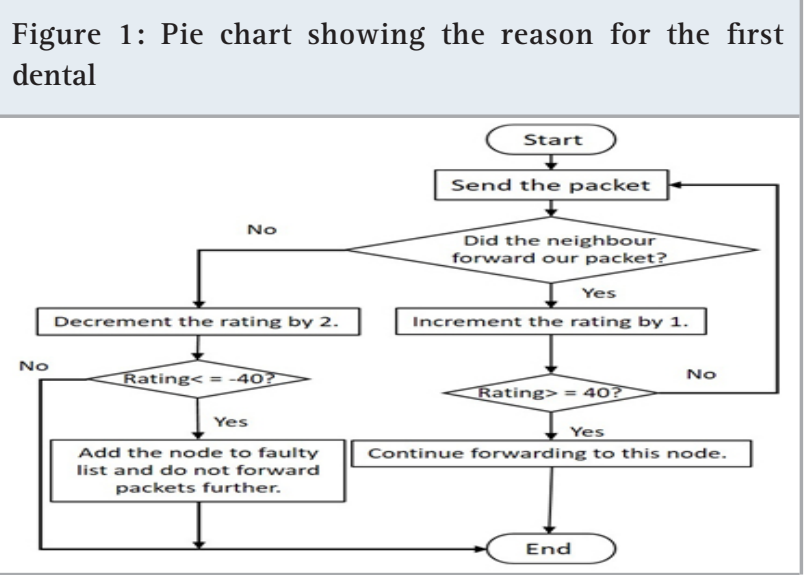

iii. Flowchart: A flowchart of the proposed method is depicted in Figure 1. The proposed method is efficient to protect the manet from multiple black hole attacks. To initialize a communication, route discovery process is carried. In the proposed work, after the success of route discovery, the source node will send packets to its neighbors. The activity of the neighbor is then examined whether it forwards or drops the packet. If the node forwards the packet, then the rating is incremented 
by 1 and if the packet is dropped then the rating is decremented by 2 . If the rating drops below -40 then that node is blacklisted and no data is further transmitted to it and if the rating reaches 40 then data is forwarded to it as usual.

\section{Simulation setup and Results}

a. Network Scenario: A mobile ad hoc network of 70 nodes having a two-ray model is shown in Figure 2. Out of 70 nodes, 50 nodes are moving freely in random motion in an area of 1000×1000 m, whereas 20 nodes have no mobility. Out of 20 fixed nodes, 10 are employed as black hole attacks. In 50 mobile nodes, first 25 nodes are transmitting the data while other nodes are receiving the data. Speed of nodes vary from $1 \mathrm{~m} / \mathrm{s}$ to $20 \mathrm{~m} / \mathrm{s}$. Each node travels at a different speed in a different direction. Each node has a transmission range of 250 metre with a packet size of 512 bytes. The Simulation time is 500 second and the traffic type is UDP.

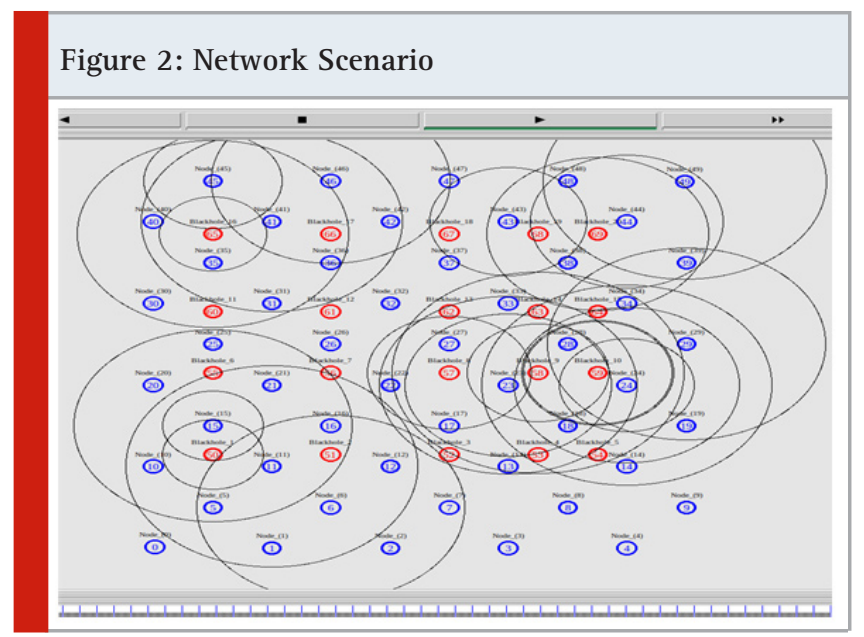

b. Results and Performance Evaluation: In this section, evaluation of a various parameter of proposed mechanism is carried and illustrated the comparison of proposed mechanism with DSR. For different number of attacks, the performance parameter like packet delivery ratio (PDR), throughput and delay are calculated. In this work, number of attacks are vary from 5 to 7 to 10 for evaluation of each parameter. From the comparison table and figure, proposed mechanism gives a significant improvement in the performance of Manet under black hole attacks can be noticed. From Figure 3 and Table 1, it
Figure 3: No. of Attackers vs. PDR

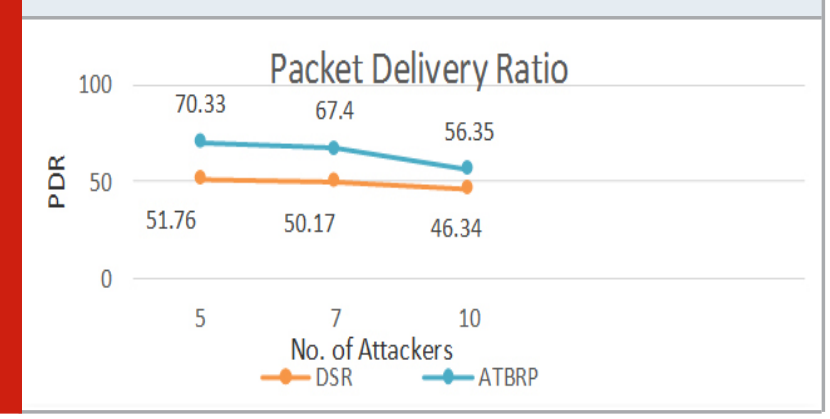

Table 1. Comparative analysis of number of attackers vs. PDR between DSR and ATBRP

\begin{tabular}{|l|c|c|c|c|c|c|}
\hline $\begin{array}{l}\text { No. of } \\
\text { Attackers }\end{array}$ & 5 & $\begin{array}{l}\text { Change } \\
(\%)\end{array}$ & $\mathbf{7}$ & $\begin{array}{c}\text { Change } \\
(\%)\end{array}$ & 10 & $\begin{array}{l}\text { Change } \\
(\%)\end{array}$ \\
\hline DSR (\%) & 51.76 & 18.57 & 50.17 & 17.23 & 46.34 & 10.01 \\
\hline ATBRP (\%) & 70.33 & & 67.4 & & 56.35 & \\
\hline
\end{tabular}

Figure 4: No. of Packets vs. Throughput

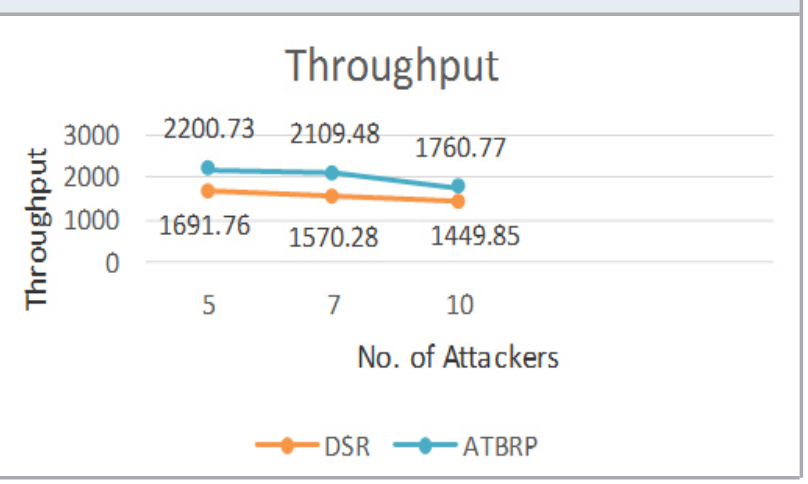

is analyzed that using DSR, the value of PDR is decreased from $51.76 \%$ to $50.17 \%$ to $46.34 \%$ if the number of attack vary from 5 to 7 to 10 , respectively. Whereas using proposed ATBRP, the value of PDR is 70.33\% to $67.4 \%$ to $56.35 \%$ if the number of attack vary from 5 to 7 to 10 , respectively. The significant improvement in PDR using proposed mechanism is depicted in Table 1 as compare to DSR.

\begin{tabular}{|c|c|c|c|c|c|c|}
\hline $\begin{array}{l}\text { No. of } \\
\text { Attackers }\end{array}$ & 5 & $\begin{array}{l}\text { Change } \\
\text { (Kbps) }\end{array}$ & 7 & $\begin{array}{l}\text { Change } \\
\text { (Kbps) }\end{array}$ & 10 & $\begin{array}{c}\text { Change } \\
\text { (Kbps) }\end{array}$ \\
\hline DSR (Kbps) & 1691.76 & 508.97 & 1570.28 & 539.2 & 1449.85 & 310.92 \\
\hline ATBRP (Kbps) & 2200.73 & & 2109.48 & & 1760.77 & \\
\hline
\end{tabular}

From Figure 4 and Table 2, it is clearly analyzed that throughput of the network using DSR is decreasing from $1691.76 \mathrm{Kbps}$ to $1570.28 \mathrm{Kbps}$ to $1449.85 \mathrm{Kbps}$ progressively with an increase in number of attacks 5 to 7 to 10 , respectively. In proposed ATBRP, the throughput goes from $2200.73 \mathrm{Kbps}$ to $2109.48 \mathrm{Kbps}$ to $1760.77 \mathrm{Kbps}$ 
for 5 to 7 to 10 attacks, respectively. The significant improvement in Throughput using proposed mechanism is depicted in Table 1 as compare to DSR.

Figure 5 and Table 3 give the relation of average delay with number of attacks.It is clear that the average end-to-end delay increases in DSR with an increase in number of attacks as compare to proposed ATBRP. For less number of attack DSR has less delay than ATBRP but with increase in attack, proposed ATBRP performs better than DSR in terms of dealy. The significant improvement in average delay using proposed mechanism for different number of attacks is depicted in Table 3 as compare to DSR.

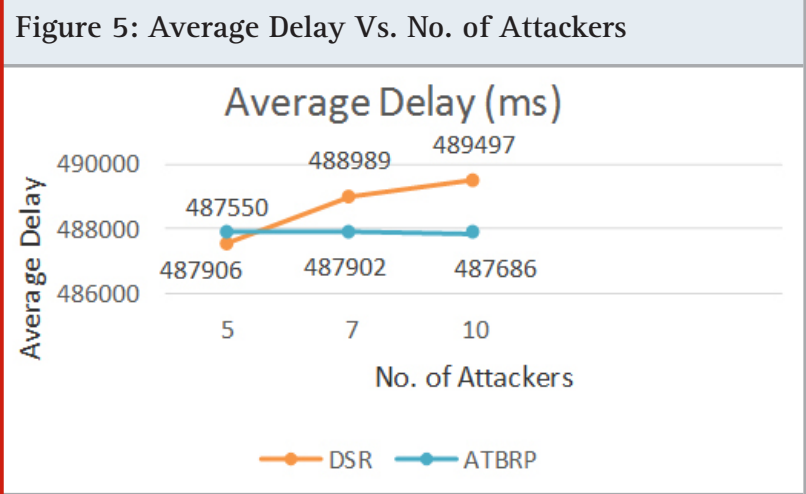

Table 3. Comparative analysis of number of attackers vsvs. Average Delay
between DSR and ATBRP
\begin{tabular}{l|c|c|c|c|c|c|}
\hline $\begin{array}{l}\text { No. of } \\
\text { Attackers }\end{array}$ & 5 & $\begin{array}{c}\text { Change } \\
\text { (ms) }\end{array}$ & 7 & $\begin{array}{c}\text { Change } \\
\text { (ms) }\end{array}$ & 10 & $\begin{array}{c}\text { Change } \\
\text { (ms) }\end{array}$ \\
\hline DSR (ms) & 487550 & -356 & 488989 & 1087 & 489497 & 1811 \\
\hline ATBRP (ms) & 487906 & & 487902 & & 487686 & \\
\hline
\end{tabular}

\section{CONCLUSION}

Security mechanism in routing protocol is very crucial to maintain quality of services and to achieve distortion less communication in ad hoc network. The well established routing protocol like DSR, AODV etc. don't have security provision to protect a Manet from active and passive attacks. The proposed trust based method yields better results in terms of throughput, packet delivery ratio and average delay for the network under multiple attacks as compare to DSR. It has been concluded that the proposed protocol using self-assessment and mutual assessment operation can improve the data transmission in the network and use trust information to protect the network. This quality of the protocol in addition to the other functions of DSR makes it more reliable and eligible than DSR in the sensitive fields where high security is required.

\section{REFERENCES}

A. A. Chavana, D. S. Kuruleb, P. U. Derec, "Performance Analysis of AODV and DSDV Routing Protocol in MANET and Modifications in AODV against Black Hole Attack," in 7th International Conference on Communication, Computing and Virtualization, vol. 79, pp. 835-844 April 2016, doi: https://doi.org/10.1016/j. procs.2016.03.108

A. H. Rushdi, "Protocol for Multiple Black Hole Attack Avoidance in Mobile Ad Hoc Networks," in Intechopen, October 2018, doi: 10.5772/intechopen.73310

A. Hammamouche, M. Omar, N. Djebari and A. Tari, "Lightweight reputation-based approach against simple and cooperative black-hole attacks for MANET," in Journal of Information Security and Applications, vol. 43, pp. 12-20, December 2018, doi: https://doi. org/10.1016/j.jisa.2018.10.004

A. Nadeem and M. P. Howarth, "A Survey of MANET Intrusion Detection \& Prevention Approaches for Network Layer Attacks," in IEEE Communication Survey \&t Tutorial, vol. 15, no. 4, pp. 2027-2045, March 2013, doi: 10.1109/SURV.2013.030713.00201

A. U. Khan, M. D. Chawhan, Y. Suryawanshi and S. Kakde, "Design of High Performance Packet Classification Architecture for Communication Networks," in Journal of Telecommunication, Electronics and Computer Engineering, vol. 9, no. 4, December 2017.

D. B. Choudhurya, L. Raghab and N. Marathe, "Implementing and improving the performance of AODV by receive reply method and securing it from Black hole attack," in International Conference on Advanced Computing Technologies and Applications, vol. 45, pp. 564-570, March 2015, doi: https://doi.org/10.1016/j. procs.2015.03.109

G. Wahane and S. Lonare, "Technique for Detection of Cooperative Black Hole Attack in MANET," in Fourth International Conference on Computing, Communications and Networking Technologies, January 2014, doi: 10.1109/ICCCNT.2013.6726621

J. Sen, S. Koilakonda and A. Ukil, "A Mechanism for Detection of Cooperative Black Hole Attack in Mobile Ad Hoc," in Second International Conference on Intelligent Systems, Modelling and Simulation, March 2011, doi: 10.1109/ISMS.2011.58

L. Tamilselvan and V. Sankaranarayanan, "Prevention of Co-operative Black Hole Attack in MANET,” Journal 
of Networks, vol. 3, no. 5, May 2008.

M. Mohanapriya and I. Krishnamurthi, "Trust Based DSR Routing Protocol for Mitigating Cooperative Black Hole Attacks in Ad Hoc Networks," in Arabian Journal for Science and Engineering, vol. 39, pp. 1825-1833 2014, doi: https://doi.org/10.1007/s13369-013-0764-1

R. J. Cai, X. J. Li and P. H. J. Chong, "An Evolutionary Self-Cooperative Trust Scheme Against Routing Disruptions in MANETs," in IEEE Transaction on Mobile Computing, vol. 18, no. 1, pp. 42-55, April 2018, doi: 10.1109/TMC.2018.2828814.

R. Kothari and D. Dembla, "Implementation of Black Hole Security Attack using Malicious Node for Enhanced-DSR Routing Protocol of MANET," in International Journal of Computer Application, vol. 64, no. 18, Febuary 2013, doi: 10.5120/10731-5504

S. Hazra and S. K. Setua, "Blackhole Attack Defending Trusted On-Demand Routing in Ad-Hoc Network," in Advanced Computing, Networking and Informatics, vol. 2, pp. 59-66, 2014, doi: https://doi.org/10.1007/978-3-
319-07350-7_7

S. S. Bhalsagar, M. D. Chawhan, Y. Suryawanshi and V. K. Taksande, "Performance Evaluation Of Routing Protocol Under Black hole Attack in Manet and Suggested Security Enhancement Mechanisms," in International Journal of Innovative Technologies and Exploring Engineering, vol. 8, no. 5, March 2019, doi: https://www.ijitee.org/wp-content/uploads/papers/v8i5/ E3214038519.pdf

Y. Wu, Y. Zhao, M. Riguidel, G. Wang and P. Yi, "Security and trust management in opportunistic networks: a survey," in Security and Communication Network, vol:8, pp. 1812-1827, September 2015, doi: https://doi. org/10.1002/sec.1116

Z. A. Zardari, J. He, N. Zhu, K. H. Mohammadani, M. S. Pathan, I. H. Muhammad and Q. M. Muhammad, "A Dual Attack Detection Technique to Identify Black and Gray Hole Attacks Using an Intrusion Detection System and a Connected Dominating Set in MANETs," in Future Internet, vol. 11, no. 3, pp. 1-17, March 2019, doi: https://doi.org/10.3390/f111030061 